\title{
Frontières
}

\section{La violence de l’objectivité}

\section{Nicolas Lévesque}

Volume 21, numéro 2, printemps 2009

Détresse psychique et antidépresseurs

URI : https://id.erudit.org/iderudit/039462ar

DOI : https://doi.org/10.7202/039462ar

Aller au sommaire du numéro

Éditeur(s)

Université du Québec à Montréal

ISSN

1180-3479 (imprimé)

1916-0976 (numérique)

Découvrir la revue

Citer cet article

Lévesque, N. (2009). La violence de l'objectivité. Frontières, 21(2), 77-79.

https://doi.org/10.7202/039462ar

Ce document est protégé par la loi sur le droit d'auteur. L'utilisation des services d'Érudit (y compris la reproduction) est assujettie à sa politique d'utilisation que vous pouvez consulter en ligne.

https://apropos.erudit.org/fr/usagers/politique-dutilisation/ 


\section{LA VIOLENCE DE L'OBJECTIVITÉ}

Nicolas Lévesque, Ph. D., psychologue en pratique privée.

Lorsque mes patients me demandent ce que je pense réellement des psychotropes, je me retiens, je ne fais pas comme leur médecin ou certaines personnes de leur entourage qui ne se gênent pas, la plupart du temps, pour leur dire de cesser sur le champ leur analyse ou leur psychothérapie, qu'ils perdent leur temps et leur argent avec moi, que la science ordonne maintenant de combiner la médication avec un traitement cognitivo-comportemental qui fait des merveilles pour faire saliver les chiens, pour dompter les phoques, les dauphins et les phobiques, pour retourner les gens au travail en moins de deux. Ce que je pense des pilules? Des lampes solaires antidépressives? Des thérapies par mouvements oculaires? Des huiles de poisson? Tout le monde aime Marineland. Tout le monde aime Marineland. Tout le monde aime Marineland.

Dans le quotidien de ma pratique, je ne fais pas la morale, je ne fais pas de prescriptions, je ne fais pas d'exposé sociophilosophique sur l'histoire de la folie et le délire matérialiste, je n'encourage pas mes patients à prendre de la médication, je ne les pousse pas non plus à refuser d'en prendre. Par contre, je me fais un devoir de mettre en contexte leur rapport aux pilules et à l'autorité qui les prescrit, sur le fond de leur histoire personnelle, de leur dynamique transférentielle et de leurs enjeux inconscients.

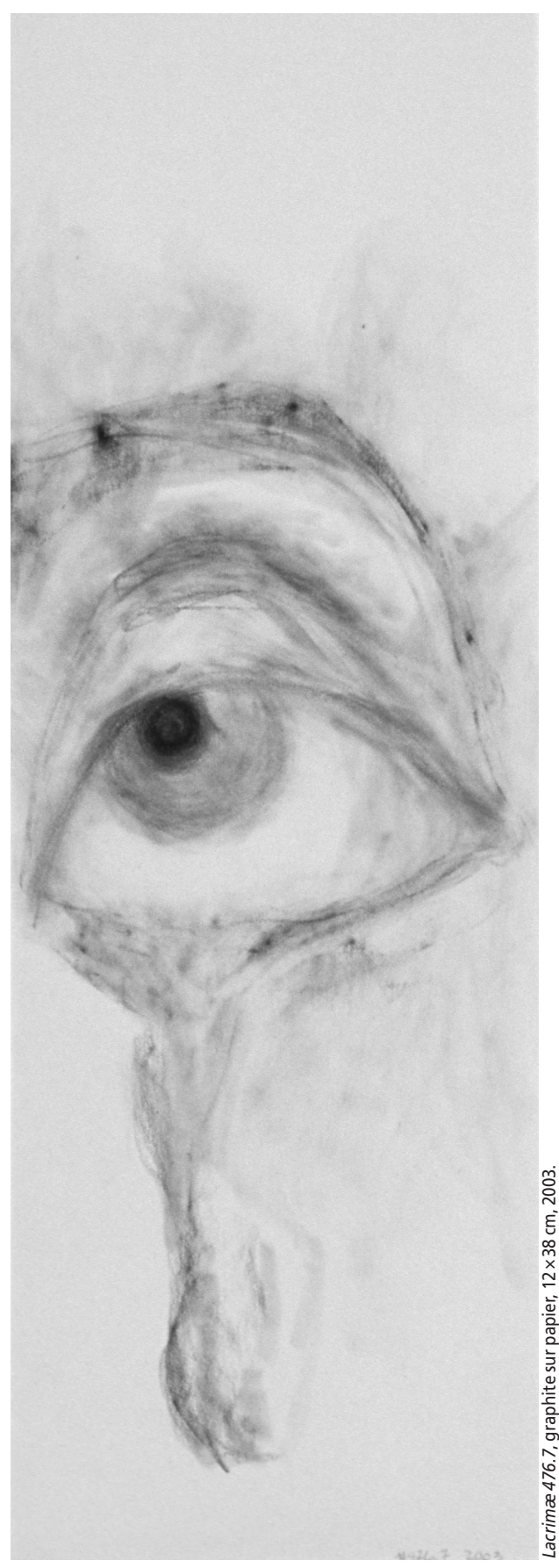

Pour l'un d'eux, il s'agissait du même drame, du même scénario d'abandon, la société répondant à sa détresse par la médication, de la même manière que ses parents, en quelque sorte, c'est-àdire d'une manière concrète, matérielle: il repartait de chez ses parents avec un chèque et quelques plats congelés, de la clinique avec ses comprimés. Personne n'était disponible sur le plan psychique et affectif pour l'écouter, porter son excès. Il lui paraissait tout à fait normal et habituel de se faire traiter ainsi pour sa dépression, puisqu'il n'avait jamais connu autre chose. Je représentais dans sa vie l'inconnu et l'on résiste toujours à l'inconnu.

Pour un autre, la dépendance à la drogue prescrite lui donnait une structure, un horaire, une ritualité dont il ressentait autant l'urgent besoin que l'incapacité de vivre cette soumission dans une relation humaine; avec les médicaments, l'ecstasy, l'alcool et les machines du gym, il semblait possible de s'abandonner, de se laisser nourrir et prendre en charge.

Sous cet angle, les pilules ressemblent à des jouets pour adultes: elles offrent la chance d'une relation avec un objet transitionnel. Dès le tout jeune âge, il devient important de ne plus afficher sa dépendance à ses parents, de la cacher sous une relation passionnelle avec sa doudou ou son toutou. De même, les médicaments permettent à mes patients de tolérer leur dépendance et leur dette envers moi. J'entends souvent les gens me dire, avec un large sourire qui trahit le message 
implicite: "C'est vraiment miraculeux ces petites pilules! Je ne me suis jamais senti aussi bien. » Je me retiens, je les laisse se défendre, je me tape moi-même dans le dos, je me dis que je ne fais pas ce métier pour qu'on me dise que je suis bon, je ne fais pas comme leur médecin qui saute habituellement sur chaque occasion de vanter les bienfaits de tel traitement ou de telle nouvelle famille de neuroleptiques.

La science aime bien les honneurs, elle est gourmande, sa soif de reconnaissance m'inquiète. Sauver des vies, trouver des remèdes au cancer, au sida, rendre les maladies moins douloureuses, les accouchements moins risqués, inventer des technologies moins polluantes, voilà en effet des réussites extraordinaires, mais pousser le fantasme de toute-puissance jusqu'à étendre l'empire scientifique au-delà des frontières de la folie, de la vie affective, de l'angoisse existentielle, du deuil, du roman familial, de la sexualité, vraiment? Doit-on vraiment subir l'appétit infini de l'enfantroi positiviste? Le fait de gaver à ce point ses sujets n'est-il donc qu'une projection de sa propre faim insatiable?

Pour l'une de mes patientes, le diagnostic et la médication qui vient avec, en pièce jointe, donnaient une réalité extérieure à sa réalité psychique; on reconnaissait enfin qu'elle était vraiment malade, qu'elle n'inventait rien, que sa souffrance était valable, qu'enfin, contrairement à ce que lui disait sa mère, elle ne se plaignait pas la bouche pleine. Pour un autre de mes patients, la dissolution du comprimé dans son corps lui donnait l'impression d'une présence intérieure, rassurante et excitante, presque orgasmique, de sa jolie psychiatre; il m'avoua que c'était comme lui faire l'amour à tous les jours, alors qu'il devait se contenter d'un rendez-vous à tous les six mois.

Un autre jeune homme n'avait jamais révélé sa tragédie. On ne lui avait jamais demandé non plus ce qui n'allait pas. Enfant hyperactif à l'école, contrôlé à grandes doses de Ritalin et de mesures disciplinaires, il n'avait jamais rencontré quelqu'un qui ait pris le temps de gagner sa confiance, de mériter le poids de son terrible secret, de son mystère à lui, le petit-fils d'un grand-père pédophile. Combien d'autres mangent leurs smarties en silence?

Assis sur les marches de l'entrée d'un centre jeunesse, ce jeune contrevenant fume une cigarette en se rappelant le visage des huit intervenants qui ont défilé cette année-là devant lui, l'un après l'autre, lui promettant d'être une personne-ressource. Il se dit à lui-même que seuls ses clopes et ses Effexor ne l'ont pas laissé tomber.

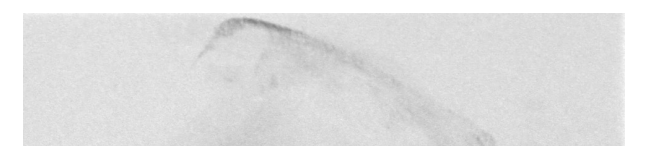

attaché à jamais à sa disparue, son unique, son irremplaçable, préférant le refus de relancer le désir au buffet dégoûtant de la manie capitaliste qui fête jour et nuit le substitut, le remplacement, les numéros, les objets échangeables; consentir à la médication, c'était consentir à ce mondelà, insensible, indifférent, machinal. Sa résistance à la médication était la seule chose qui le gardait en vie, la seule lutte qui gardait le canon dirigé vers l'extérieur. Il a eu beaucoup de problèmes avec sa compagnie d'assurances qui a coupé les versements, car il aurait semblé que monsieur ne se montrait pas coopératif dans sa volonté de retourner au travail. Artaud a raison: il y a des suicidés de la société.

Je n'avais pas vraiment le temps d'écrire ce texte, mais je me suis dit qu'il était temps de réfléchir à l'impact de ce manque de temps généralisé. On le sait bien, le réseau de la santé, tout comme celui de l'éducation, ne dispose pas des ressources nécessaires pour permettre à ses employés de bien faire leur travail, ce qui est impossible lorsque le temps manque toujours, les listes d'attente s'allongent, les classes deviennent de plus en plus nombreuses et les exigences bureaucratiques des institutions de plus en plus encombrantes. Tout se passe comme si la structure avait pour but d'empêcher qu'un événement singulier survienne entre un intervenant et un patient ou entre un professeur et un élève. Au lieu de faire cet aveu d'impuissance, les institutions se complaisent dans le déni, le contrôle et l'illusion de toute-puissance. Au lieu de tout mettre en œuvre pour accueillir et faire advenir, au cœur même de la structure, la singularité - c'est-à-dire précisément sa limite, son horizon, ce qui lui échappe, à la fois son ombre et son ڤ̊̀ désir -, le discours officiel tend toujours Eં à justifier l'état des choses; si les choses sont ainsi, c'est qu'elles doivent l'être. C'est ainsi que l'idéologie neuro-pharmacopsychiatrique vient justifier l'ordre actuel et installer la pratique de la prescription des psychotropes comme la justification idéale du bien-fondé du système en place. On ne peut que rester muet devant cette pirouette rhétorique: si l'on manque de temps et de compétence pour écouter l'histoire personnelle qui se dissimule dans le symptôme, ce n'est pas, au fond, parce que l'on manque de temps, de formation ou que l'argent n'est pas redistribué de manière adéquate dans le réseau de la santé, mais bien parce que le discours singulier du patient n'a aucune valeur en dehors de ce qu'il est nécessaire de savoir pour poser un diagnostic et rendre possible la classification et la prescription. Les patients n'ont plus à parler, car on s'occupe de leurs neurotransmetteurs qui eux révèlent tout, 
disent tout, c'est-à-dire rien, surtout rien d'autre que ce que veut leur faire dire le ventriloque de l'idéologie au pouvoir.

Que ce soit par le biais de la religion ou de la science, il est fascinant d'observer comment l'ordre social produit sans cesse un discours immunitaire, une idéologie permanente d'autojustification qui donne une légitimité à l'exercice de son pouvoir. Dans notre illusion démocratique, nous croyons qu'il existe d'abord des idées, un débat, puis qu'ensuite une série de normes et de règles sont établies à partir de ce discours. Or, les relations entre le savoir et le pouvoir sont plutôt à concevoir dans l'autre sens, c'est-à-dire qu'il y aurait d'abord une réalité politique, un jeu de forces qui impose des hiérarchies, une structure sociale, et ensuite un savoir qui vient justifier les dispositifs du pouvoir, afin de le maintenir, de masquer ses injustices et de lui fournir une légitimité idéologique. Autrement dit, dans le cas qui nous concerne, le discours neuro-pharmacopsychiatrique n'est pas omniprésent parce qu'il touche une vérité ou un avancement des connaissances, mais bien parce qu'il participe à une légitimation du pouvoir en place, prenant ainsi le relais du discours religieux qui, dans l'histoire de la folie, a produit les fondements qui ont assuré l'impunité de la violence et de l'exclusion.

Comment comprendre alors la popularité incroyable des psychotropes? C'est pourtant simple, le transfert s'élance toujours vers l'objet-supposé-pouvoir, tel que Lacan aurait pu le formuler après avoir entendu ce que Foucault et Althusser ont essayé de dire à la psychanalyse - tel que je le formule après avoir reçu d'une patiente un poème intitulé L'objet-supposé-pouvoir.

En chacun de nous gît ce fantasme ultime de la soumission à quelqu'un ou quelque chose de tout-puissant, ce rêve du sentiment océanique de l'abandon total à cette instance qui connaît le chemin, détient les réponses et a le pouvoir de nous protéger. C'est, bien entendu, le rêve de retourner flotter avec insouciance dans le ventre d'une mère parfaite, d'une mère forteresse, ce qui, en d'autres termes, incarne le souhait d'éviter tout travail de deuil, toute épreuve de l'altérité. C'est humain, trop humain. Le toxicomane d'État est donc maintenu à l'état du nourrisson qui ressent chaque goutte de lait comme un cadeau du Tout-Puissant, ce que le petit humain a la chance de revivre en recevant son pain quotidien, la drogue de Mom ou celle du bon docteur, le représentant sur terre de Mère Nature et de Père Pharma. Dans la scène de la prescription de pilules, c'est la science qui, en réalité, invite les brebis égarées à entrer dans son ventre géant se mettre à l'abri, offrant ainsi une sécurité par le sacrifice de la liberté. En pleine tempête, en haute mer, qui ne rêve pas de se réfugier dans le ventre de la baleine? C'est ce qu'ont compris tous les pouvoirs totalitaires. Mais rassurez-vous, rien de cela en démocratie

Il est presque risible de voir dans les psychotropes un progrès, en les comparant aux chaînes, aux saignées, aux lobotomies, de la même manière que l'on perçoit les armes chimiques et nucléaires comme un progrès par rapport aux canons et aux baïonnettes. Le progrès, ce serait donc le déploiement de la violence à distance, un remote control qui se fait dans la précision, le calme et la propreté, ce qui permet de ne pas faire l'expérience de la souffrance et de ne même plus avoir à s'en laver les mains. Je suis évidemment contre tous les 8000 traitements par électrochocs qu'ont subis l'année dernière, au Québec, nos étrangers intérieurs, qui n'ont pas d'ONU pour les défendre, mais ces méthodes ont toutefois le mérite de ne pas cacher la violence du traitement.

Nous ne vivons qu'un nouveau chapitre de la même histoire, l'histoire de la domination de la raison sur la déraison, de la mesure sur la démesure; il s'agit toujours de contrôler le corps, le lieu du mal à éradiquer, et d'étouffer l'excès de la passion associée au féminin. Nous revivons la scène grecque de l'exclusion du pathos, sous l'autorité du logos, enfermé dans cet exil domestique, ce dehors intérieur que représente la scène tragique. Aujourd'hui, l'excès d'émotions est bien contenu dans ces marges que sont les scènes artistiques qui, en cas de débordement, sont remplacées par les asiles modernes; il est permis au citoyen-spectateur de vivre par procuration, par une sorte de catharsis, toutes les émotions, les excès, les démesures et les transgressions, mais il lui est interdit de vivre cette expérience en son nom, dans la cité, en dehors des limites de la scène du spectacle; à ce manque de retenue répond la camisole chimique. On comprend donc la nécessité de dépeindre, à grand tirage de mauvaises presses, les artisans du spectacle perpétuel comme des victimes de leurs excès, des gens à qui l'on ne voudrait surtout pas ressembler dans la vie quotidienne, des étoiles si loin du «vrai monde », condamnés à l'exil pour avoir osé défier les lois divines.

Étrangement, la plupart des médecins et psychiatres que je connais sont animés des meilleures volontés qui soient, d'un réel désir de porter secours, d'une sensibilité à la souffrance d'autrui. Quiconque a une expérience de la folie sait qu'il faut mettre une limite, que c'est même là la demande même de la folie. Nul discours adolescent ici, pas de rêve naïf de laisser courir libre- ment la folie, ce qui revient à l'abandonner. La question est plutôt celle-ci: s'il faut, en effet, une limite, pourquoi mettre une limite chimique? À quel pouvoir s'adresse le cri de la folie? N'est-ce pas son drame qu'elle répète ainsi en rencontrant toujours le même type de pouvoir, c'est-àdire cette violence qui dans sa perversion utilise l'autre comme son objet, éclipsant du coup son altérité, sa singularité, son existence même? Sans s'en rendre compte, le pouvoir psychiatre est le prisonnier du scénario de la folie, jouant inconsciemment le rôle qui lui est donné, au lieu d'analyser cette dynamique transférentielle, de lui résister et de lui offrir une nouvelle voie.

Dans son délire des grandeurs, sa soif de conquérir tous les continents, son souhait de ne rencontrer aucune limite, ni résistance, la science est devenue folle, elle renvoie à la folie un miroir de son absence de limite, de son évitement de l'épreuve du deuil, de l'épreuve de l'autre. Elle n'aide pas la folie, elle la mime et dans cette confusion classique et typique des relations psychotiques fusionnelles, on ne sait plus trop qui est qui, qui est dans qui, qui a avalé l'autre; est-ce la science qui a avalé la folie ou la folie qui a avalé la science? Et s'il est vrai qu'à la folie une limite doit répondre, qui, en retour, se charge de mettre une limite à la raison? 